NATIONAL LABORATORY

MANAGED BY UT-BATTELLE

FOR THE DEPARTMENT OF ENERGY

\title{
Measurements and Analysis of Mock Holdup Configurations Using Fast-Neutron Imaging
}

\section{September 2012}

\section{Prepared by}

Paul Hausladen Matthew Blackston

Felix Liang Jason Newby

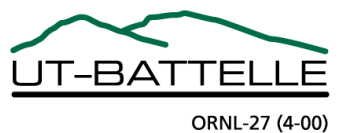




\section{DOCUMENT AVAILABILITY}

Reports produced after January 1, 1996, are generally available free via the U.S. Department of Energy (DOE) Information Bridge.

Web site http://www.osti.gov/bridge

Reports produced before January 1, 1996, may be purchased by members of the public from the following source.

National Technical Information Service

5285 Port Royal Road

Springfield, VA 22161

Telephone 703-605-6000 (1-800-553-6847)

TDD 703-487-4639

Fax 703-605-6900

E-mail info@ntis.gov

Web site http://www.ntis.gov/support/ordernowabout.htm

Reports are available to DOE employees, DOE contractors, Energy Technology Data Exchange (ETDE) representatives, and International Nuclear Information System (INIS) representatives from the following source.

Office of Scientific and Technical Information

P.O. Box 62

Oak Ridge, TN 37831

Telephone 865-576-8401

Fax 865-576-5728

E-mail reports@osti.gov

Web site http://www.osti.gov/contact.html

This report was prepared as an account of work sponsored by an agency of the United States Government. Neither the United States Government nor any agency thereof, nor any of their employees, makes any warranty, express or implied, or assumes any legal liability or responsibility for the accuracy, completeness, or usefulness of any information, apparatus, product, or process disclosed, or represents that its use would not infringe privately owned rights. Reference herein to any specific commercial product, process, or service by trade name, trademark, manufacturer, or otherwise, does not necessarily constitute or imply its endorsement, recommendation, or favoring by the United States Government or any agency thereof. The views and opinions of authors expressed herein do not necessarily state or reflect those of the United States Government or any agency thereof. 


\title{
MEASUREMENTS AND ANALYSIS OF MOCK HOLDUP CONFIGURATIONS USING FAST-NEUTRON IMAGING
}

Paul Hausladen, Matthew Blackston, Felix Liang, and Jason Newby

Date Published: September 2012

\author{
Prepared by \\ OAK RIDGE NATIONAL LABORATORY \\ Oak Ridge, Tennessee 37831-6283 \\ managed by \\ UT-BATTELLE, LLC \\ for the \\ U.S. DEPARTMENT OF ENERGY \\ under contract DE-AC05-00OR22725
}





\section{CONTENTS}

\section{Page}

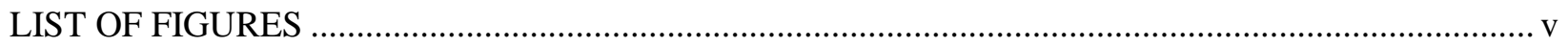

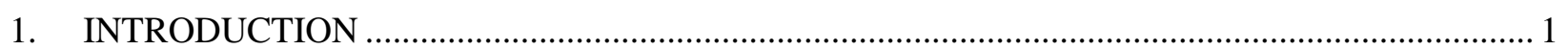

2. DESCRIPTION OF THE FAST-NEUTRON IMAGER ......................................................... 1

3. SOURCES OF ERROR IN INFERRED SOURCE INTENSITY ............................................... 3

4. MEASUREMENTS OF MOCK HOLDUP CONFIGURATIONS ................................................ 7

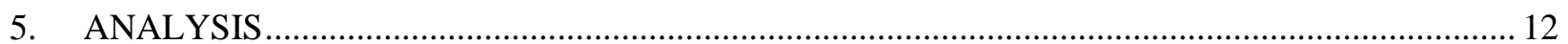

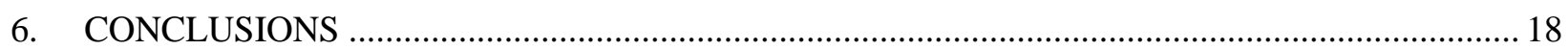

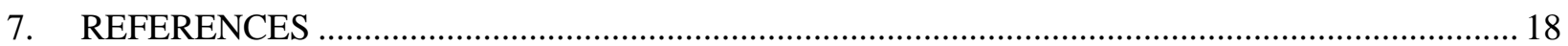





\section{LIST OF FIGURES}

1 The prototype coded-aperture fast-neutron imager. ....................................................... 2

2 Efficiency variations across the face of the neutron detector. .............................................. 4

3 Schematic diagram of the source-mask-detector geometry of the coded-aperture technique showing the inherent sensitivity of the technique for measuring distance to

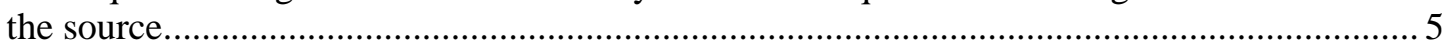

4 Schematic diagram of the parallax between two measurements being used to determine

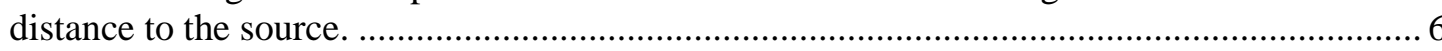

5 Schematic diagram of the worst-case distributed source geometry where the average

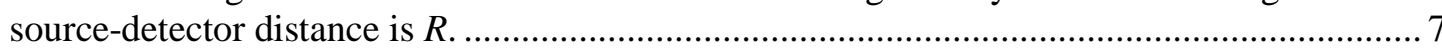

6 Photographs of the large L-duct and HEPA filter house used for mock holdup

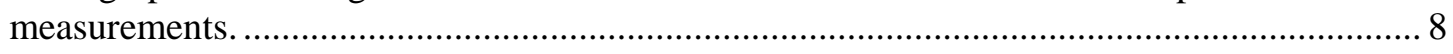

$7 \quad$ The HEPA filter box surrogate and the ${ }^{252} \mathrm{Cf}$ button sources. ............................................ 9

8 Three representative imaging measurements for the zoomed imager configuration............... 10

9 Comparison of wide-angle and zoomed neutron images of an example neutron source

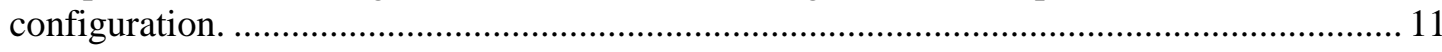

10 Photographs of the large L-Duct where the statistical significance of the neutron source

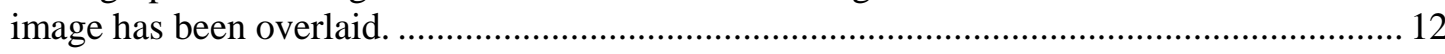

11 Illustration of non-source pixel fluctuations in an image induced by error in distance to

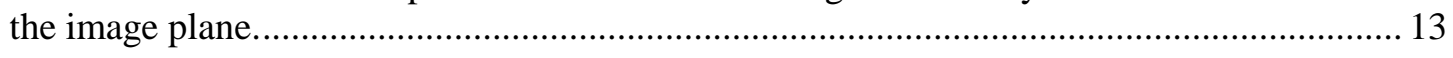

12 Extraction of the net systematic error per image count. ................................................. 14

13 The calculated net systematic error per image count for two configurations......................... 14

14 Comparison of net systematic error per image count for a point source and an extended

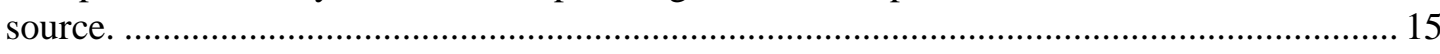

15 Expected distance resolution for the zoomed point source and extended source configurations as a function of image counts.

16 Expected uncertainty in source strength for the zoomed point source and extended source configurations as a function of mass of $\mathrm{PuO}_{2}$.

17 Net systematic error per image count for two configurations of sources in the large LDuct plotted (left) as a function of assumed distance and (right) as a function of the size of the mask projection on the detector. 



\section{INTRODUCTION}

The present report investigates the use of fast-neutron imaging for performing quantitative measurements of plutonium holdup at fuel-cycle facilities. In particular, initial experimental assessment of the performance and limitations of fast-neutron imaging for this purpose has been made by analyzing measurements of a series of mock holdup configurations. These mock holdup configurations were constructed by placing known-intensity ${ }^{252} \mathrm{Cf}$ sources in representative fixtures, including a duct and a filter house, that correspond to potential holdup configurations at fuel cycle facilities and can readily be investigated in the laboratory. The present report was prepared in fulfillment of milestone M2FT12OR0402042, "Complete proof-of-concept imaging measurements to quantify SNM in holdup configurations" of Fuel Cycle R\&D Material Protection, Accounting, and Control Technology (MPACT) work package FT-12OR040204, "Fast-Neutron Imaging to Quantify Nuclear Materials."

The present effort to assess fast-neutron imaging for the purpose of quantifying plutonium holdup was instigated by the recent development of a prototype fast-neutron imager by Oak Ridge National Laboratory (ORNL). The imager records images of the distribution and intensity of fast-neutron sources such as plutonium via their fast-neutron emissions. Fast-neutron imaging is an appealing mode of measurement in scenarios where contact measurements with non-imaging detectors may be impractical or their results ambiguous because of surrounding material or nearby sources. The use of fast neutrons (as opposed to the more numerous gamma rays) is appealing because of their ability to penetrate equipment and nuclear material that is opaque to gamma radiation. In addition to images, quantitative measurements of neutron sources are possible in those instances where the distance to the source of neutrons is known or can be measured. In instances where neutron emanations are sufficiently numerous, neutron measurements may be better able to give quantitative mass estimates of holdup than gamma-ray measurements.

The remainder of the report is organized as follows. First, a brief description of the imager is provided suitable to enable a discussion of sources of error. Then, a number of sources of error are identified for quantitative neutron imaging measurements. In particular, systematic error in the estimation of holdup quantities due to the shape and inferred distance to a holdup deposit are considered in detail. Then, the measurements are described. Last of all, the set of mock holdup measurements are analyzed and errors in inferred source strength estimated.

\section{DESCRIPTION OF THE FAST-NEUTRON IMAGER}

In this section, a brief description of the imager is provided with sufficient detail to enable a discussion of errors in neutron source strength inferred from imaging measurements.

The imager consists of a position-sensitive fast-neutron detector panel and a high-density polyethylene (HDPE) aperture that spatially modulates neutrons incident on the detector. The neutron detector portion of the imager consists of a panel of 16 individual detectors that pack closely together to give a total area of $42.8 \times 42.8 \mathrm{~cm}^{2}$ and comprise 1600 individual pixels in a $40 \times 40$ grid. The sensitive volume of the detectors consists of a total of 8 liters of liquid scintillator EJ-309, an organic proton recoil scintillator which enables neutron-gamma discrimination via pulse shape. All organic scintillators are sensitive to neutrons and gamma rays, so neutron-gamma discrimination is essential for performing quantitative measurements of neutron source strengths. Details of the design and construction of the neutron detector are reported elsewhere [1].

The aperture portion of the imager consists of a HDPE coded aperture having a base-19 modified uniformly redundant array (MURA) pattern [2]. The aperture thickness is determined by stacking between one and four identical $2.4 \mathrm{~cm}$ thick masks to the desired thickness, normally as thick as possible without 
undue collimation. For the measurements presented in this report, the size of the mask pattern was based on $1.4 \mathrm{~cm}$ apertures. The angular field of view of the imager is set by adjusting the distance between the neutron detector panel and the aperture from a minimum of $23 \mathrm{~cm}$ to a maximum of $110 \mathrm{~cm}$. The field of view of the imager is determined by the opening angle of the $19 \times 19$ element mask base pattern from the center of the detector panel. For the pattern based on $1.4 \mathrm{~cm}$ apertures used in the present work, the field of view ranges between 13.8 and 60 degrees. A photograph of the prototype fast-neutron imager is shown in Fig. 1, where the polyethylene aperture is visible to the right of the photograph and the neutron detector panel visible to the left.

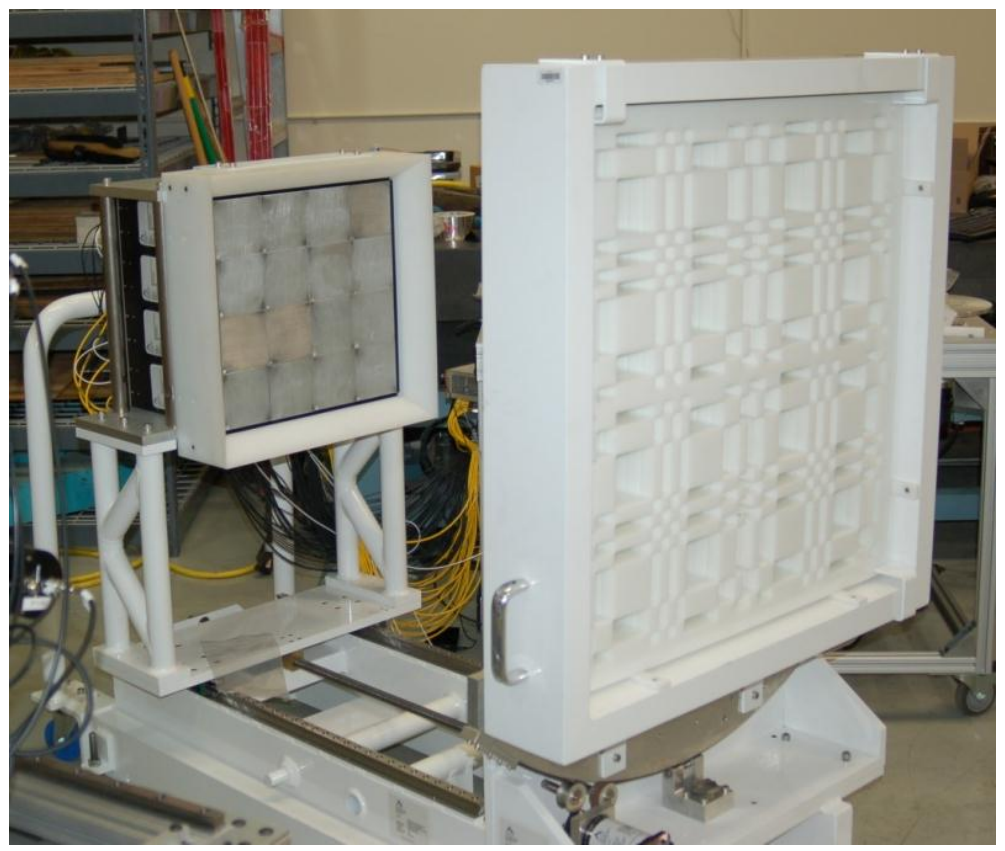

Fig. 1. The prototype coded-aperture fast-neutron imager.

A coded aperture is essentially a set of multiplexed pinholes in which each individual pinhole casts an image of the neutron sources in the field of view on the detector panel. The pattern of pinholes is specially chosen so that the image can be uniquely decoded from the combined shadows of the aperture cast by the sources on the detector panel. For a point source, multiplexing pinholes in this way increases signal-tonoise over a single pinhole by the square root of the number of mask openings, in this case by a factor of approximately 13. For a base-19 MURA, there are $19^{2}$ unique (and orthogonal) aperture shadow patterns that can be cast on the detector panel, each corresponding to a basis direction. Consequently, the position resolution corresponds to approximately 1/19th the field of view. In the case of a $2 \mathrm{~m}$ field of view at the object plane, that resolution corresponds to just over $10 \mathrm{~cm}$. The amount of neutron source attributable to a particular basis direction can be computed by adding the neutron detector counts behind each portion of the detector that would be directly illuminated from that direction and subtracting from each portion of the detector that would be obscured. An image can be thought of as the projection of the data along each of the basis directions. For the base-19 MURA, the positions of the closed and open portions of the mask can be interchanged by rotating the mask by 90 degrees. By measuring in both of these configurations (referred to as "mask" and "antimask" configurations, respectively), background counts that have not been modulated by the mask but may not be uniform across the detector can be eliminated. In the present imager, rotation between mask and antimask configurations has been automated.

The intrinsic efficiency of the neutron detector for ${ }^{252} \mathrm{Cf}$ fission neutrons as used for the imaging measurements in the present work was 0.20 . Using a stack of three $2.4 \mathrm{~cm}$ thick masks for a total 
thickness of $7.2 \mathrm{~cm}$, the portions of the detector in the shadow of closed portions of the mask still count approximately 0.32 of the incident neutrons, corresponding to an effective mask thickness of 1.14 mean free paths. In such a configuration, in the limit where all counts are coming from a source in the field of view, the ratio of signal counts to total counts is approximately 0.52 . In the case where only a singlethickness $2.4 \mathrm{~cm}$ thick mask is used, the portions of the detector in the shadow of closed portions of the mask count approximately 0.61 of the incident neutrons, corresponding to an effective mask thickness of 0.5 mean free paths. Note that the effective thickness of the mask is not linear in the actual thickness of the mask. This nonlinearity originates mainly from neutron scattering in the detector lessening the effective contrast of the mask shadow.

\section{SOURCES OF ERROR IN INFERRED SOURCE INTENSITY}

For neutron imaging measurements, as with all counting measurements, a number of factors can contribute to errors in inferring the neutron source intensity and plutonium mass of a holdup deposit from the result of the imaging measurement. Some of these factors relate to the holdup itself. For instance, the holdup geometry may be incompletely known. The shape of the holdup, distance to the holdup, and selfattenuation of the holdup can all contribute to errors in attribution of source strength. By using fast neutrons, the self-attenuation of holdup deposits are minimized (compared to gamma rays), but not eliminated. Moreover, the neutron rate must be related to a plutonium mass. For this purpose, the isotopics of the holdup deposit must be known by other means, such as a gamma-ray measurement, a destructive assay measurement, or process knowledge. In the present work, the errors in inferred source strength originating from shape and distance will be considered.

Some of the factors that contribute to errors in inferring the neutron source intensity relate to the detector. For instance, the efficiency of the detector must be accurately known at the time of the measurement for the appropriate neutron energy spectrum. Often, sources for calibration consist of ${ }^{252} \mathrm{Cf}$ fission neutron sources whose energy spectrum contains more energetic neutrons than the spectrum of energies produced by $\mathrm{PuO}_{2}$. Gain changes may be observed due to drifts in high voltage or temperature. Organic scintillators exhibiting pulse-shape discrimination also exhibit changes in pulse shape with temperature. Unaccounted for changes in pulse shape may result in reduced counting of neutrons or the inclusion of additional gamma rays misidentified as neutrons. The quality of pulse-shape discrimination is also a function of rate. Consequently, measuring neutron sources whose gamma-ray activities are very disparate may introduce biases towards overcounting sources with unusually high gamma activity. In a complicated instrument having 1600 pixels, detector nonuniformity that has been incompletely characterized can lead to error. In the present work, the errors in inferred source strength originating from incompletely characterized detector nonuniformity will be examined.

Last of all, all counting measurements have errors associated with counting statistics. Statistical errors must always be considered. Given that the mask is half open, and that the closed portions of the mask transmit the fraction $T$ of incident neutrons, the signal rate in the detector is $0.5 s_{0} \varepsilon \Omega(1-T)$, where $s_{0}$ is the source neutron rate and $\Omega$ is the solid angle of the detector (as a fraction of $4 \pi$ steradians). For codedaperture imagers, the error in each pixel is the square root of the total counts in the detector. If the source is entirely in one pixel of the reconstructed image, the relative error in the signal after a time $t$ due to counting statistics alone is

$$
\sigma=\frac{\sqrt{\left(b_{0}+0.5 s_{0} \varepsilon \Omega(1+T)\right) t}}{0.5 s_{0} \varepsilon \Omega(1-T) t}
$$


where $b_{0}$ is the background rate in the detector from all sources other than the target source $s_{0}$. If the source is distributed over $n_{p}$ pixels, the error in the inferred signal strength is increased by the factor $\sqrt{n_{p}}$.

While there is an efficiency $\varepsilon$ for the composite detector, each constituent pixel may (and typically does) have a slightly different efficiency than the average. These different pixel efficiencies are measured by removing the mask and nearly uniformly illuminating the detector with neutrons from a source (the expected residual $1 / r^{2}$ dependence is removed given the known source-to-detector distance). Such an efficiency map is shown in the left panel of Fig. 2. In this efficiency map, the 16 individual detectors can be identified by eye owing to the lower efficiency of the edge and corner pixels in each detector.
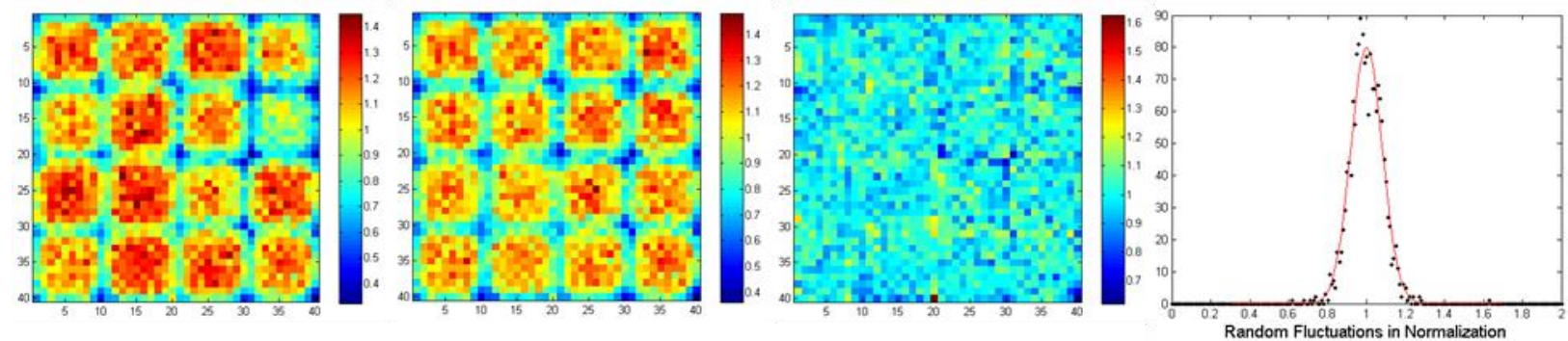

Fig. 2. Efficiency variations across the face of the neutron detector. From left to right, the raw efficiency map, after correction for the average efficiency of each detector, and after correction for the average position dependence within a detector. The remaining fluctuations, shown histogrammed in the rightmost plot, are randomly distributed about unity with a standard deviation of 0.08 .

Does the uncertainty in this efficiency map contribute significantly to the uncertainty of counting measurements? In fact, it does not. This efficiency map consists primarily of an average detector response that, for each detector, has slightly higher or lower efficiency. In order to demonstrate this, in the leftcenter panel of Fig. 2, the efficiency map is shown divided by the average efficiency of each detector. In the right-center panel, each detector is divided again by the average position dependence of counts on the detector. The remaining panel has random variations in pixel gains about unity with a standard deviation of 0.08 , consistent with 250,000 total counts in the detector. Because all pixels contribute to an imaging measurement, the error in each pixel of an imaging measurement due to pixel-to-pixel variations in gain will be $0.2 \%$. This error is small compared to other errors and need not be considered.

For holdup that may be localized within large industrialized equipment, the uncertainty in source strength due to the uncertainty in distance to the source can be large. For a given number of neutron counts in the detector, the source strength responsible for the counts is proportional to the distance to the source squared, $R^{2}$. As a consequence, the relative error in the inferred source strength $\Delta s / s$ due to error in radius $\Delta R$ is

$$
\frac{\Delta s}{S}=2\left(\frac{\Delta R}{R}\right)
$$

Typical scales of equipment under inspections might range from some tens of centimeters to a meter or more. For reasonable distances from the source to the detector, the error in plutonium mass could be $20 \%$ or more from distance alone. This error is significant. Likewise, there can be significant reduction in error by measuring the effective distance to holdup.

The coded-aperture technique has some inherent sensitivity to distance imparted by measuring the size of the projection of the mask pattern onto the neutron detector panel. This sensitivity is shown schematically 
in Fig. 3, where two potential source positions separated by $\Delta d$ at a distance $d$ from the mask cast mask shadows on the neutron detector whose size differs by $\Delta x$.
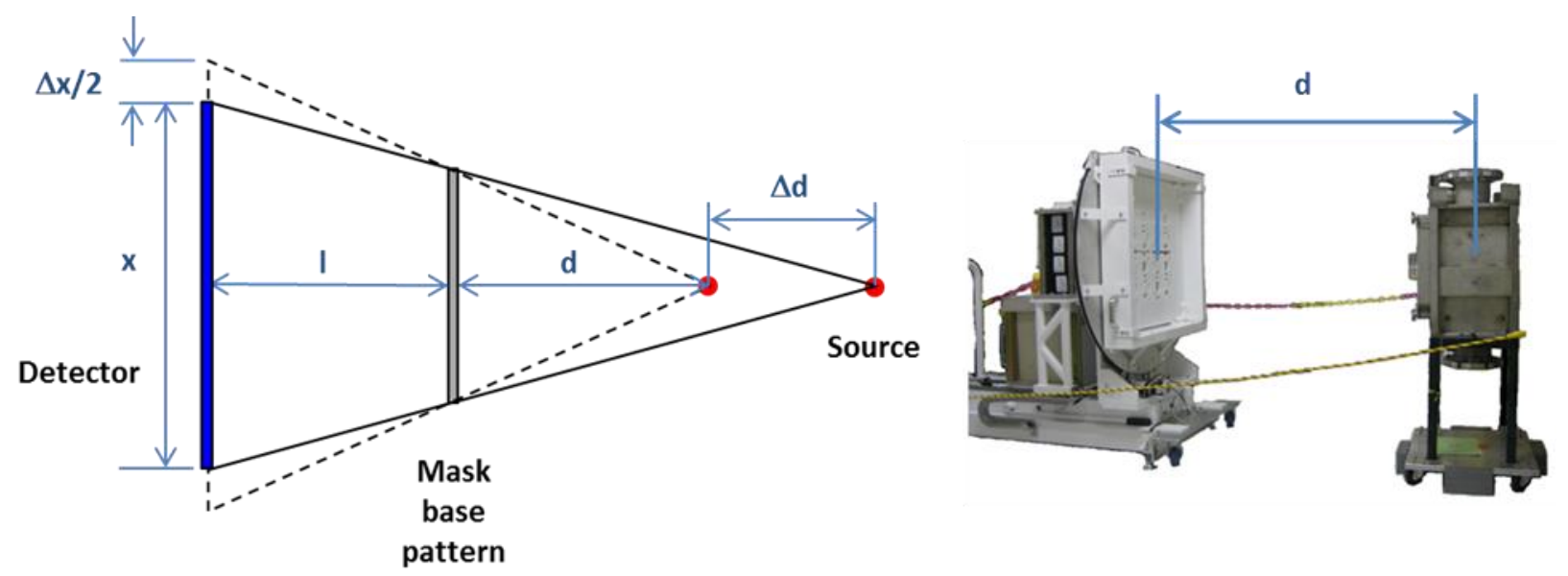

Fig. 3. Schematic diagram of the source-mask-detector geometry of the coded-aperture technique showing the inherent sensitivity of the technique for measuring distance to the source.

For this setup, the relative uncertainty in the source-to-mask distance $d$ is related to the relative uncertainty in size of the shadow of the mask base pattern by

$$
\frac{\Delta d}{d}=\left(\frac{d+l}{l}\right) \frac{\Delta x}{x}
$$

where $l$ is the mask-to-detector distance and the consequent relative uncertainty in the source strength is

$$
\frac{\Delta s}{s}=2\left(\frac{\Delta R}{R}\right)=2\left(\frac{d}{l+d}\right) \frac{\Delta d}{d}=2\left(\frac{d}{l}\right) \frac{\Delta x}{x} .
$$

Note that the most favorable ratio of $d / l$ is unity, but a more reasonable value (particularly when desiring a compact instrument) is 5. As a result, the uncertainty in source strength is somewhere between 2 and 10 times the uncertainty in size of the mask shadow on the detector. The largest the mask shadow can be is the full detector size of 40 pixels. If the size of the shadow can be determined to a single pixel out of 40 , then the error in inferred source strength due to uncertainty in distance will be somewhere between 5\% and $25 \%$, depending on the configuration of the imager. If the size of the mask shadow can be determined to a tenth of a pixel out of 40, then the error in inferred source strength due to uncertainty in distance will be somewhere between $0.5 \%$ and $2.5 \%$. The accuracy to which the size of the mask shadow can be determined will be discussed in detail during the analysis section.

Another method of measuring distance is to use parallax between two successive measurements where the imager was repositioned a known amount between measurements. Using this method, shown schematically in Fig. 4, the position of the image two neutron sources separated by $\Delta d$ at a distance $d$ from the mask will move a distance $x$ and separate by a distance by $\Delta x$. In the present work, no measurements of this variety were performed, but the method has been analyzed because it has a potential for much more accurate distance determination. 


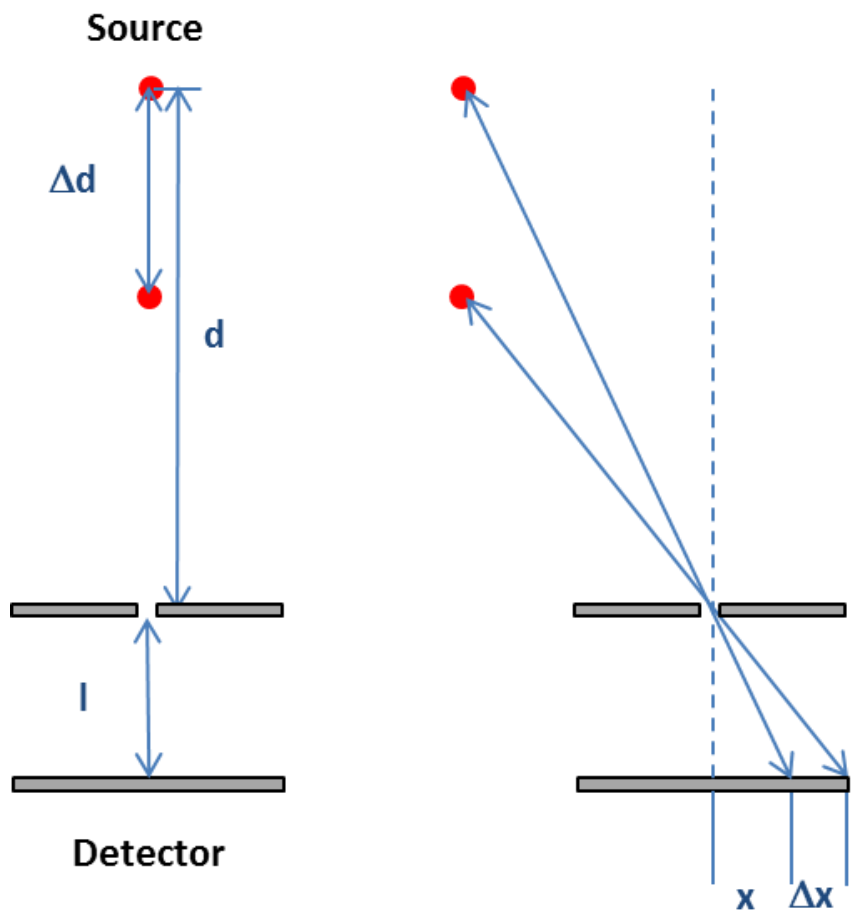

Fig. 4. Schematic diagram of the parallax between two measurements being used to determine distance to the source.

For this setup, the relative uncertainty in the source-to-mask distance $d$ is related to the relative motion of the two source images by:

$$
\frac{\Delta d}{d}=\frac{\Delta x / x}{1+\Delta x / x}
$$

where $x$ is the distance the source moves on the image. The consequent relative uncertainty in the source strength is

$$
\frac{\Delta s}{s}=2\left(\frac{\Delta R}{R}\right)=2\left(\frac{d}{l+d}\right) \frac{\Delta d}{d}=2\left(\frac{d}{l+d}\right)\left(\frac{\Delta x / x}{1+\Delta x / x}\right) .
$$

For this setup, supposing the position of the source in the image is known to 1 pixel and it has been moved half the field of view (20 pixels), the uncertainty in source strength for the same range of imager configurations considered earlier is now between 5\% and 8\%, a much better range than for the method using mask shadow size. Furthermore, determination of the centroid of a source is easily done to much better than a single pixel with modest statistics. If the position of the source can be determined to a tenth of a pixel, the contribution to the source uncertainty from the uncertainty in distance will be less than $1 \%$.

In assigning a single distance to what is likely to be a spatial distribution of neutron-emitting material, the effect of the shape of the source on the inferred source strength has been neglected. A quick calculation can show that, in the limit of no self-attenuation, this omission is justified. Suppose there is a distributed neutron source whose average position is a distance $R$ from the detector. Since the imager can identify the distribution of the neutron source in the imaging plane, it is enough to consider a source that is distributed 
in distance from the detector. Considering only source distributions whose average position is $R$ and whose extent is at most $\Delta R$, the largest change in detected count rate will be if the source is split into half at $R-\Delta R$ and half at $R+\Delta R$. This configuration is shown schematically in Fig. 5.

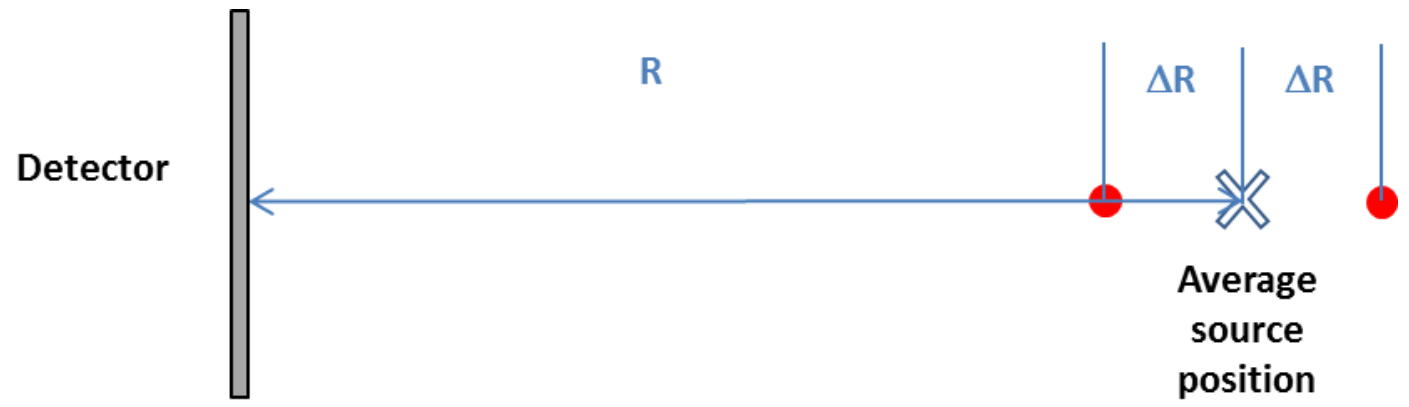

Fig. 5. Schematic diagram of the worst-case distributed source geometry where the average source-detector distance is $R$.

Supposing a source of strength $S_{0}$ at distance $R$ from the detector will record $N_{0}$ counts. Then, the split source will record

$$
N_{0}\left(1+3\left(\frac{\Delta R}{R}\right)^{2}\right)
$$

counts. If one is capable of attributing these counts to an count-weighted average distance, they will be attributed to the distance:

$$
R^{\prime}=R\left(1-2\left(\frac{\Delta R}{R}\right)^{2}\right)
$$

As a result, the source strength that will be attributed to the source will be

$$
S=S_{0}\left(1-\left(\frac{\Delta R}{R}\right)^{2}\right) .
$$

This change in attributed source strength is minor compared to other errors. For instance, at a distance of $2 \mathrm{~m}$, a piece of equipment having a full depth of $40 \mathrm{~cm}$ would only add an error of $1 \%$ due to uncertainty in the shape of a holdup deposit.

\section{MEASUREMENTS OF MOCK HOLDUP CONFIGURATIONS}

A series of mock holdup measurements were performed using the fast-neutron imager. A number of ducts, pipes, junctions, and a HEPA house are used as training aids in the ORNL Safeguards Laboratory. Two of these fixtures, the HEPA filter house and the large L-duct, were chosen as exemplars of fuel-cycle facility equipment for use with the mock holdup measurements. Photographs of the (left) L-duct and (right) HEPA filter house are shown in Fig. 6. 


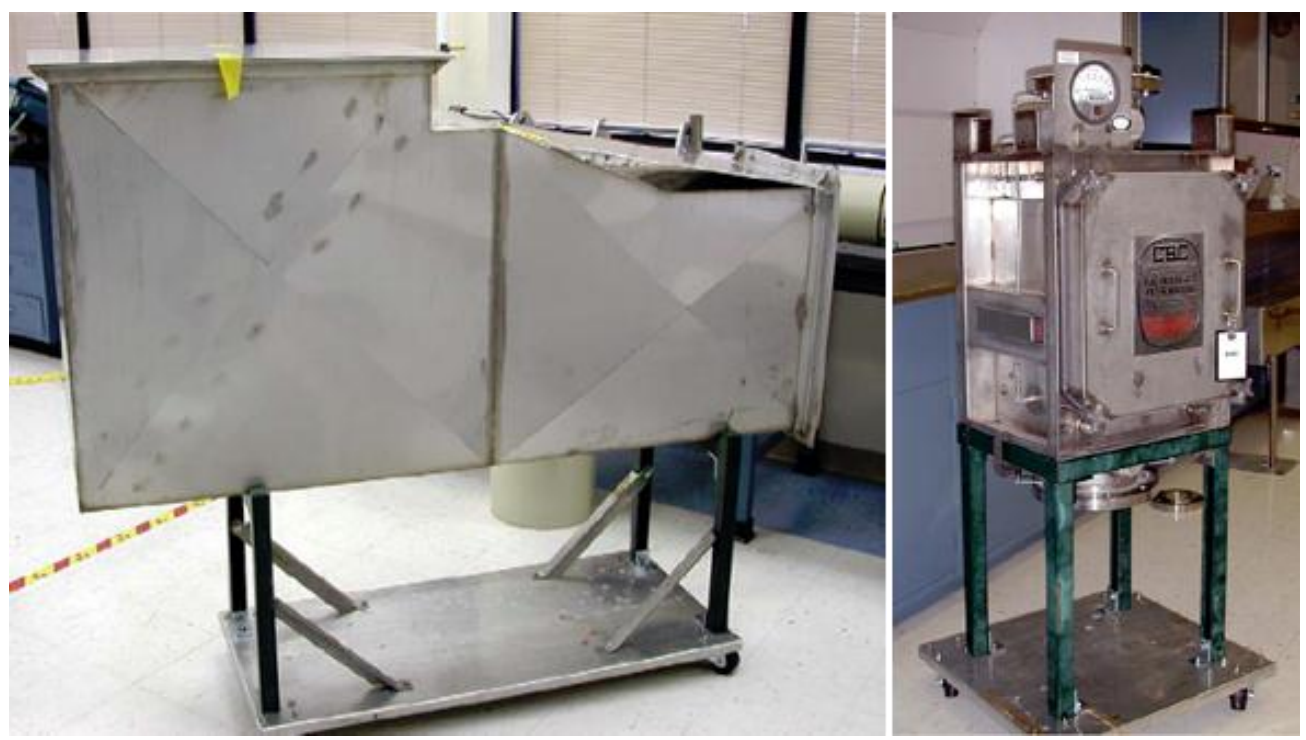

Fig. 6. Photographs of the large L-duct and HEPA filter house used for mock holdup measurements.

Holdup configurations were mimicked by placing known-intensity ${ }^{252} \mathrm{Cf}$ sources in the fixtures. For the present work, five identical ${ }^{252} \mathrm{Cf}$ sources were used. Each of the sources consists of a $0.25 \mathrm{in}$. diameter by $0.5 \mathrm{in}$. long pellet having an activity of $7 \mu \mathrm{Ci}$ (as of May 2012) for a neutron emission rate of 30,000 per second. $\mathrm{PuO}_{2}$ from typical commercial reactor fuel has a neutron emission rate of approximately 650 neutrons per second per gram (approximately 20 neutrons per second per gram per exposure (in $\mathrm{GWd} / \mathrm{MTU})$ ) [adapted from 3,4]. For this activity level, each of the ${ }^{252} \mathrm{Cf}$ sources is equivalent to the neutron source strength of $46 \mathrm{~g}$ commercial reactor-grade $\mathrm{PuO}_{2}$. In this conversion, no correction is made for different neutron detection efficiencies that might result from the differences in emitted neutron energies between $\mathrm{PuO}_{2}$ and ${ }^{252} \mathrm{Cf}$.

The HEPA filter house, as its name suggests, houses a HEPA filter cartridge that is $30 \mathrm{~cm}$ on a side and seals within the filter house. For the purposes of the mock holdup measurements, a copy of the cartridge box was constructed from plywood. The surrogate cartridge box had slots for positioning each of three $28 \mathrm{~cm} \times 28 \mathrm{~cm} \times 0.22 \mathrm{~cm}$ aluminum plates on which the ${ }^{252} \mathrm{Cf}$ sources were mounted as desired using double-sided tape. In this way, the position and extent of the neutron source could be varied to test the ability of the imager to determine the neutron source strength under a variety of conditions. One source configuration (the "point source" configuration) consisted of all five sources mounted in the middle of one aluminum plate. By changing which slot the source was inserted in the surrogate cartridge box, different source-to-imager distances could be measured. Likewise, a second configuration consisted of the five sources distributed on a single aluminum plate. Two more configurations consisted of the five neutron sources distributed among three aluminum plates in order to distribute the sources across the imaging plane as well as in distance from the imager. Figure 7 shows a photograph of (left) the filter box beside the surrogate filter box and (middle) a close-up photograph of the surrogate filter box in which the position of the aluminum plates is visible. The three positions shown in the photograph were the only slots used in the present work. A close-up of the five ${ }^{252} \mathrm{Cf}$ sources mounted via double-sided tape is shown in photograph on the right. 

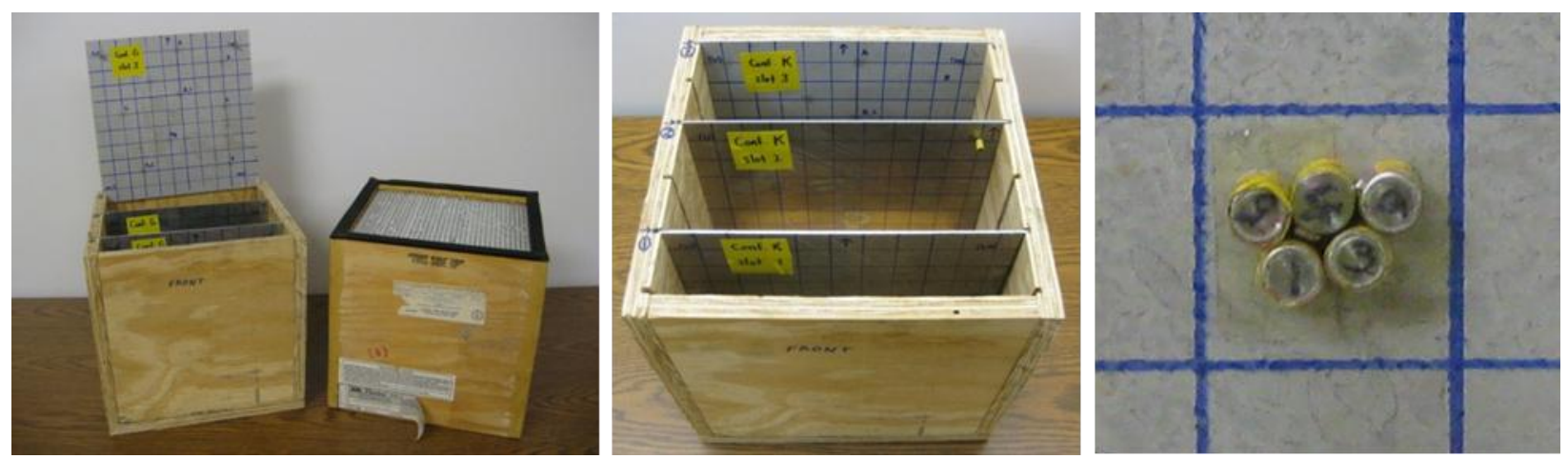

Fig. 7. The HEPA filter box surrogate and the ${ }^{252} \mathrm{Cf}$ button sources. (Left) the surrogate filter box is shown next to the HEPA filter, (center) the three aluminum plates show the positions used for source placement in the present work, and (right) the five ${ }^{252} \mathrm{Cf}$ sources shown mounted to one plate.

For the imaging measurements of the HEPA house, two general imager configurations were used. In the first configuration, the imager was set up for a wide field of view. In this configuration, the imager was positioned so that the distance between the center of the $2.4 \mathrm{~cm}$ thick mask and the center of the HEPA house measured $167.5 \mathrm{~cm}$. Mask-detector separations, depending on the measurement, ranged between the minimum of 22.8 and $33.1 \mathrm{~cm}$. In this configuration, the distance from the center of the mask to the three slots in the surrogate filter box measured 159.2,170.2, and $180.2 \mathrm{~cm}$. The field of view for the wide-angle configuration ranged between 2 and $3 \mathrm{~m}$. In the second configuration, the imager was set up for a zoomed field of view. For these measurements, the position of the imager was not changed, but the addition of two $2.4 \mathrm{~cm}$ mask thicknesses (for a total of $7.2 \mathrm{~cm}$ ) decreased the distance from the center of the mask to the three slots in the filter box to 156.8 .167 .8 , and $177.8 \mathrm{~cm}$. The smaller field of view, ranging between 60 and $80 \mathrm{~cm}$ at the position of the sources, was accomplished by pulling the detector back to mask-detector separations of $85.5,97.5$, or $109.5 \mathrm{~cm}$. Each measurement corresponded to 1 hour of acquisition, half of which was in the mask configuration and the other half of which was in the antimask configuration. Three representative imaging measurements for the zoomed imager configuration are shown in Fig. 8.

For the example measurements in the figure, the top row shows the reconstructed images in units of neutron counts. In the middle row, schematic diagrams showing the corresponding configuration of sources on the three aluminum plates in the surrogate filter box are shown. In locations where more than one neutron source has been positioned, the number of sources at that position is indicated parenthetically. Note that in these examples, one of the source configurations is pointlike while two are extended in the image plane as well as in depth. Next, the three images are shown where the color axis indicates statistical significance (in standard deviations) and overlaid on a photograph of the HEPA house. In the color scale, all points within two standard deviations of zero activity are clear and show through to the HEPA house photograph. Note that the combination of the neutron emission intensity overlaid on the photograph would already be manifestly useful in quantifying holdup.

Most source configurations were recorded in both wide-angle and zoomed neutron images. In Fig. 9, the wide-angle and zoomed images are compared for one example configuration. Above, the raw neutron images, having a color scale indicating neutron counts, are shown. Below, those images are again converted so that the color scale indicates statistical significance and overlaid on the photograph with the appropriate distance scale. 


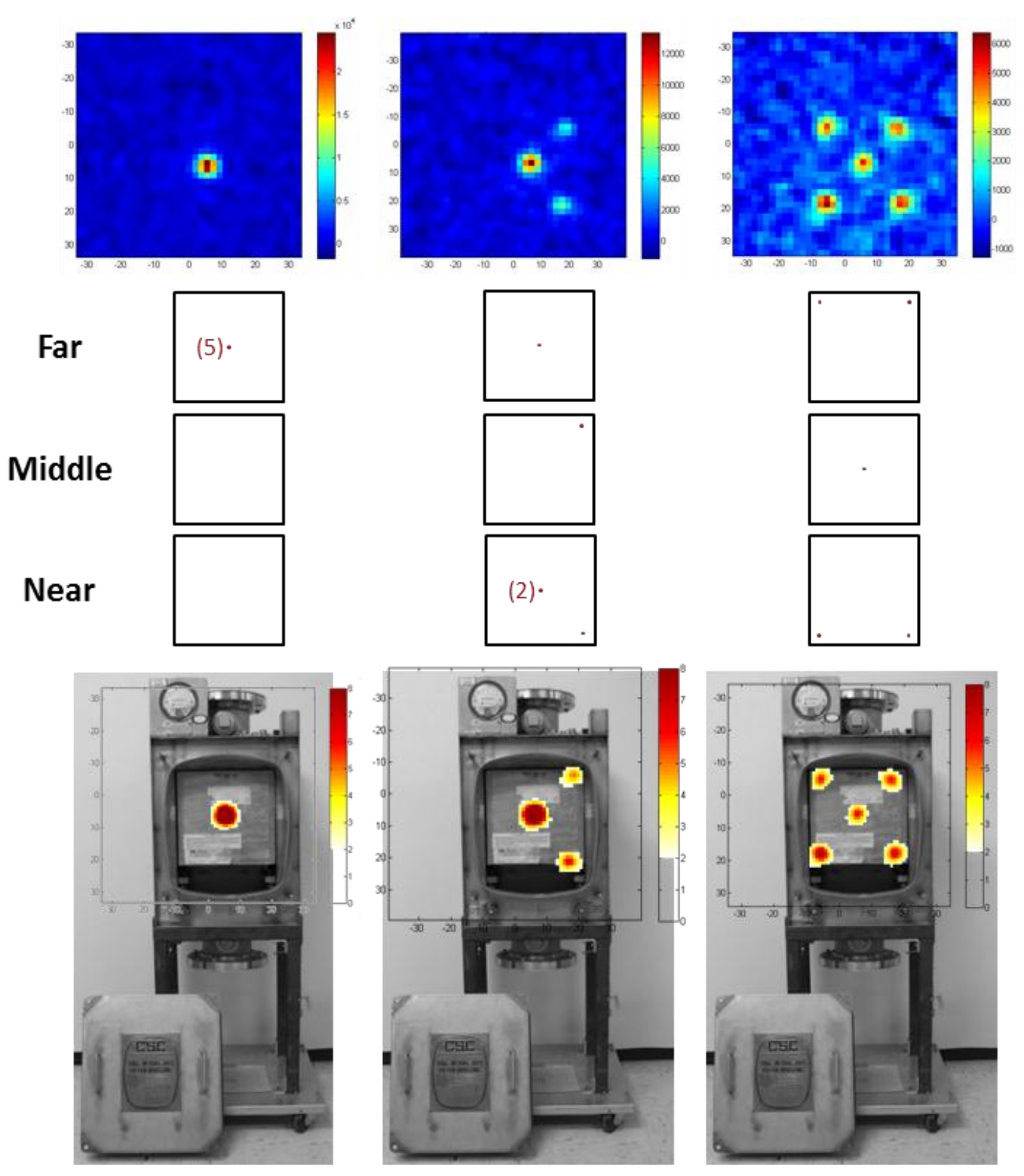

Fig. 8. Three representative imaging measurements for the zoomed imager configuration. From top to bottom are shown the reconstructed neutron image, a schematic diagram of the source configuration, and a photograph of the HEPA house on which the statistical significance of the radiation measurement is overlaid. 


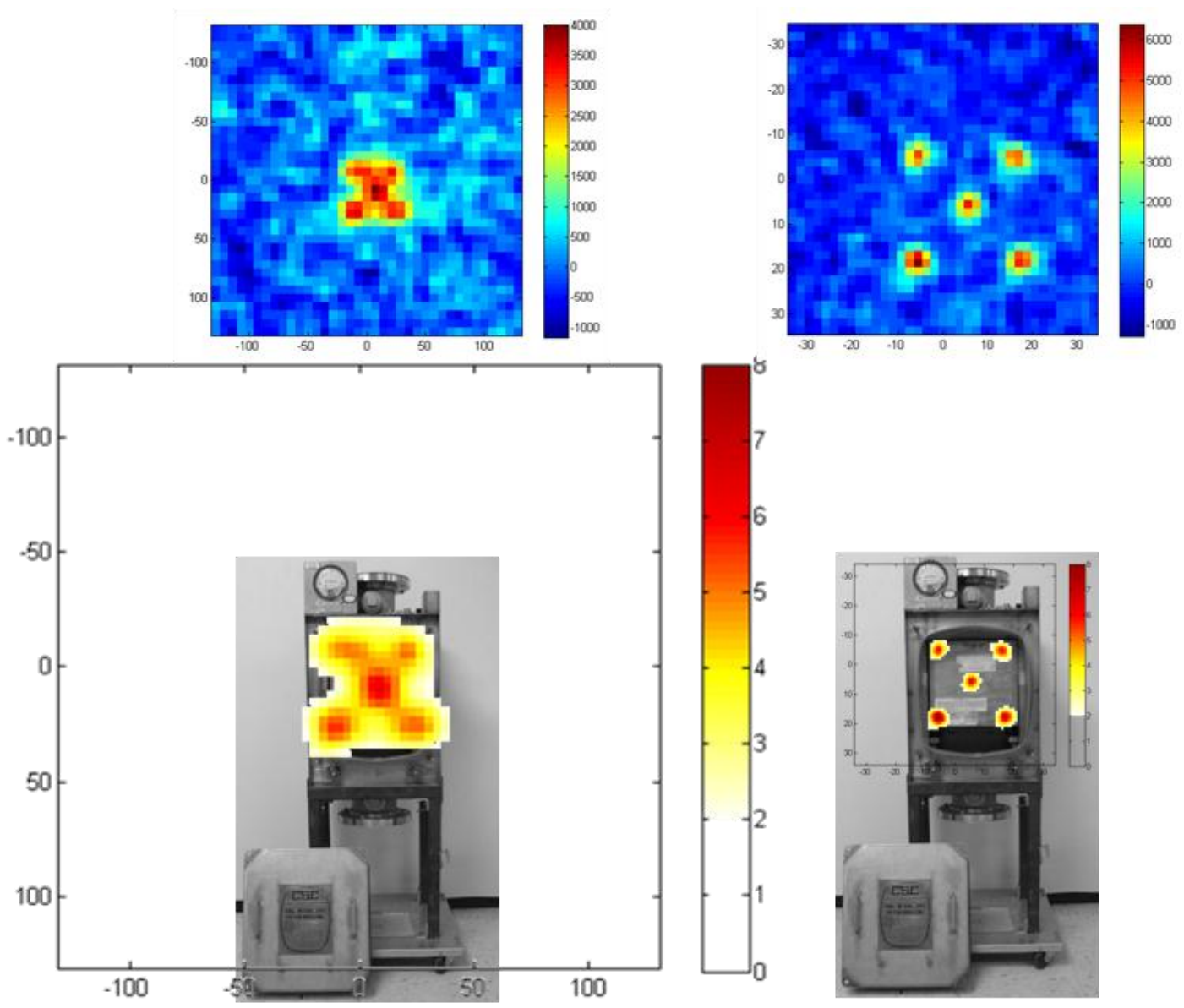

Fig. 9. Comparison of wide-angle and zoomed neutron images of an example neutron source configuration. Above, the reconstructed neutron images are shown. Below, the image is converted to statistical significance and laid over a photograph of the HEPA filter house. The axes indicate the field of view of the neutron imager.

Note that the axes indicate the field of view of the imager. Good spatial resolution is possible in a zoomed image of the HEPA house because it is small, only about $45 \mathrm{~cm}$ in width. In contrast, the large L-duct is roughly five times larger. At that size, only a wide-angle configuration is possible if it is desirable to keep the entire duct in the field of view.

For the large L-duct, two source configurations were measured. Each of these measurements also corresponded to an hour of acquisition. In the first configuration, the sources were distributed in the imaging plane as well as in depth. In the second, the sources were distributed in a single plane. These two source configurations are shown in Fig. 10, where again the color axis indicates the statistical significance of the neutron source intensity (in standard deviations) and it has been laid over on a photograph of the L-duct. As before, all image points where the reconstructed intensity is within two standard deviations of zero are clear and show through to the L-duct photograph.

Note that in both images there are five ${ }^{252} \mathrm{Cf}$ sources. The image on the left appears to show six sources because one source coincides with the edge of the field of view where the repetition of the aperture pattern cannot distinguish between a source leaving one side of the image or entering the other. 

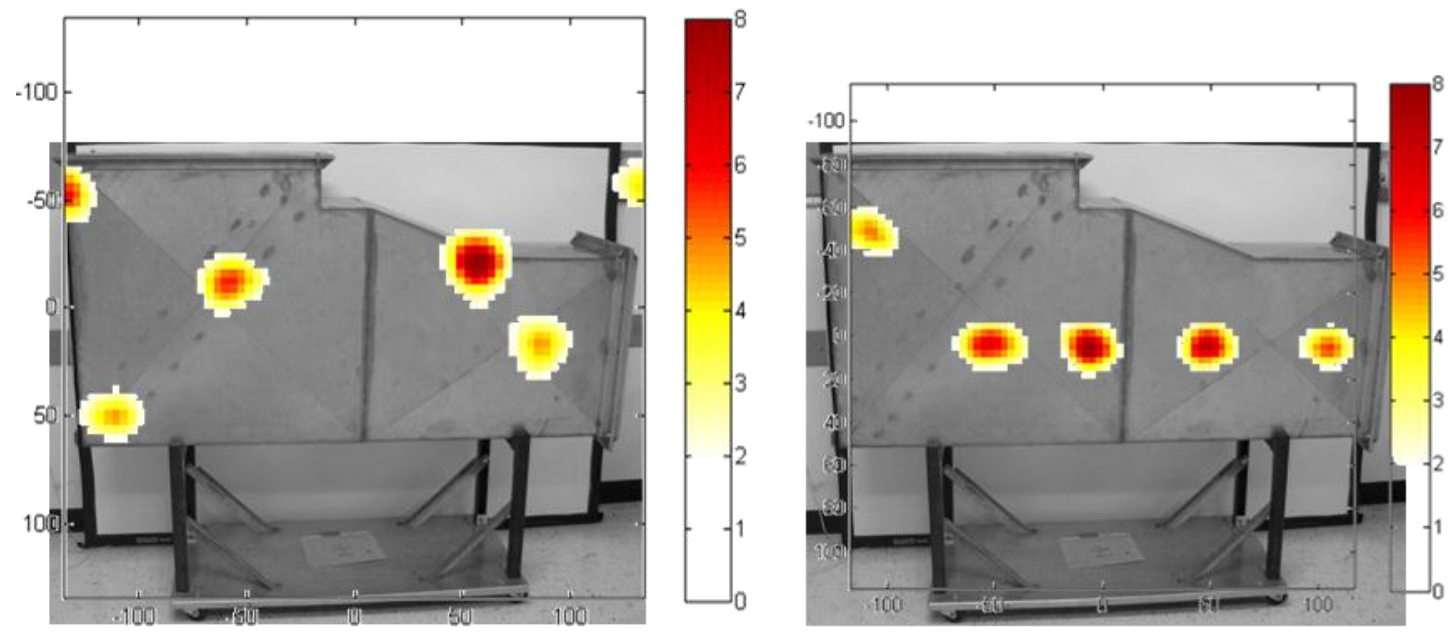

Fig. 10. Photographs of the large L-Duct where the statistical significance of the neutron source image has been overlaid.

\section{ANALYSIS}

The goal of the analysis was to understand how well the distance to a source or source distribution could be measured based on the inherent sensitivity to distance of the coded aperture technique imparted by measuring the size of the projection of the mask pattern onto the neutron detector panel. Furthermore, it is desirable to know how the accuracy of this distance measurement scales with the number of recorded source counts. That way, the error in inferred source strength due to errors in localization for sources with strengths corresponding to anticipated quantities of holdup can be predicted. In addition to being able to scale anticipated performance to appropriate source strengths, it is also desirable to understand the effect of a distribution of material, both in the imaging plane and in depth, on the ability to determine distance. As a result, measurements will be presented for point sources and extended sources. While the accuracy of distance measurements presented in this work are in some cases excellent, it is not expected that these measurements will be representative of holdup measurements generally. This section ends by considering improved distance measurement using parallax from successive measurements where the imager has been moved in between. While no measurements were performed using this technique, it should improve source distance determination so that statistical errors will dominate, even when scaling to realistic source strengths.

For a general source configuration, identifying the size of the projection of the mask pattern on the source is a complicated proposition. Fortunately, this process can be performed more easily by reconstructing the image at a number of presumed distances. At the correct distance, the root-mean-square (RMS) fluctuations in the value of non-source pixels should be close to statistical, that is, the square root of the total counts in the detector. For an arbitrary distance, fluctuations in the value of non-source pixels originate from counting statistics and also from systematic contributions proportional to the number of image counts. This can be written

$$
R M S_{t o t}=\sigma_{e x p}=\sqrt{\left(\alpha N_{I}\right)^{2}+N_{T}}
$$

where $\boldsymbol{R} \boldsymbol{M} \boldsymbol{S}_{\text {tot }}$ corresponds to the measured fluctuations, $\boldsymbol{N}_{\boldsymbol{T}}$ to the total counts in the detector, and $\boldsymbol{N}_{\boldsymbol{I}}$ to the number of imaged counts. The systematic contribution to fluctuations of non-source pixels proportional to image counts originates from loss of orthogonality of the basis patterns when 
reconstructing using the incorrect pattern size. The value $\boldsymbol{\alpha}$ corresponds to this contribution, and it is minimized at the correct distance. The above equation can be solved for $\boldsymbol{\alpha}$ :

$$
\alpha=\frac{1}{N_{I}} \sqrt{\sigma_{e x p}^{2}-N_{T}}
$$

This can be illustrated for a measurement of a point source. It is useful to begin discussion with a measurement that has the most inherent sensitivity to distance. For this purpose, measurement of a point source where the mask-to-detector distance $\boldsymbol{l}$ is as large as possible (compared to the source-to-mask distance $\boldsymbol{d}$ ) is desired. In the "zoomed" measurement of the point source shown in the previous section, the source-to-mask distance was $177.8 \mathrm{~cm}$ and the mask-to-detector distance was $109.5 \mathrm{~cm}$. A total of 180,000 neutron counts were recorded, leading to an imaged total of 60,000 counts. This measurement was reconstructed assuming source-to-mask distances ranging from 143 to $206 \mathrm{~cm}$. In Fig. 11, reconstructed images are shown for (A), $145 \mathrm{~cm},(\mathrm{~B}), 175 \mathrm{~cm}$, and (C), $201 \mathrm{~cm}$. The larger RMS fluctuations in non-source pixels are apparent in the images. Furthermore, the RMS fluctuations are plotted as a function of assumed distance $\boldsymbol{d}$ in the far right panel of Fig. 11.
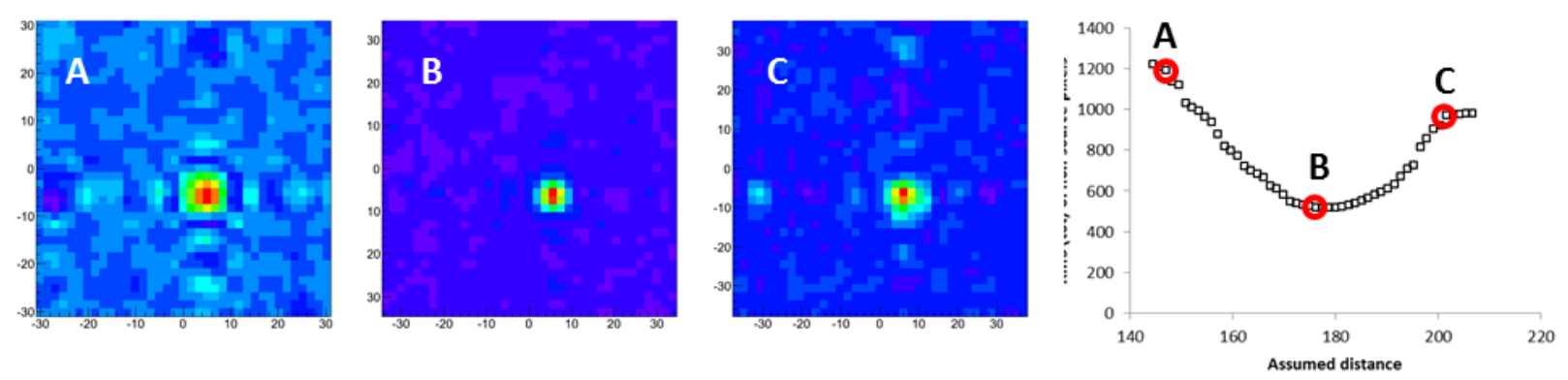

Fig. 11. Illustration of non-source pixel fluctuations in an image induced by error in distance to the image plane. From left to right, the reconstructed images for assumed distances of (A) $145 \mathrm{~cm}$, (B) $175 \mathrm{~cm}$, and (C) $201 \mathrm{~cm}$ are shown along with (far right) a plot of the RMS fluctuations in non-source pixels as a function of assumed distance showing a minimum for the correct distance.

For this measurement, the systematic contribution to the RMS fluctuations can be extracted. The value $\boldsymbol{\alpha}$ is simply this systematic contribution per image count. On the left-hand side of Fig. 12, the total RMS fluctuations, statistical contributions, and net systematic contributions are shown for the above pointsource data. On the right-hand side of Fig. 12, this quantity is normalized to yield systematic fluctuations per image count.

The expected systematic contributions per image count can be calculated simply by simulating perfect mask projections onto the detector panel and then reconstructing images for a number of assumed distances. For perfect data, we would expect there to be maximum sensitivity to changes in the assumed distance because there is no systematic error when the assumed distance matches the true distance. Such a case is shown in the blue curve in the plot on the left-hand side of Fig. 13. However, actual images never reaches zero systematic error and so have less sensitivity to distance. The is due to a number of reasons, including the discrete pixelization of the detector, the thickness of the mask and detector, scattering in the detector, and collimation and scattering in the mask. The discrete pixelization of the detector contributes systematic error in the reconstruction when the pixel boundaries do not line up with the projection of the mask pattern. This is true for almost all source-to-mask distances at a given mask-to-detector distance, including the source-to-mask distance corresponding to the above point source measurement. The expected systematic error per image count for this source-to-mask distance is shown in the blue curve in the plot on the right-hand side of Fig. 13. 

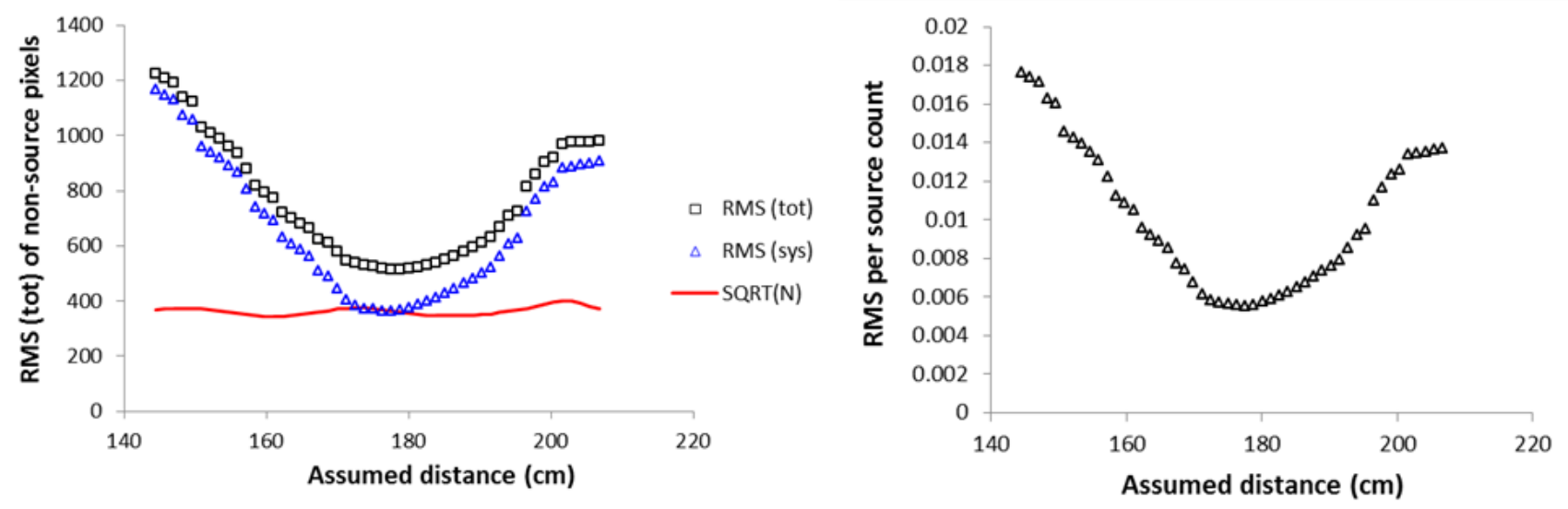

Fig. 12. Extraction of the net systematic error per image count. Left, the total RMS fluctuations as plotted as a function of assumed distance along with the statistical contribution and deduced net systematic contributions. Right, this net systematic error is normalized per image count.
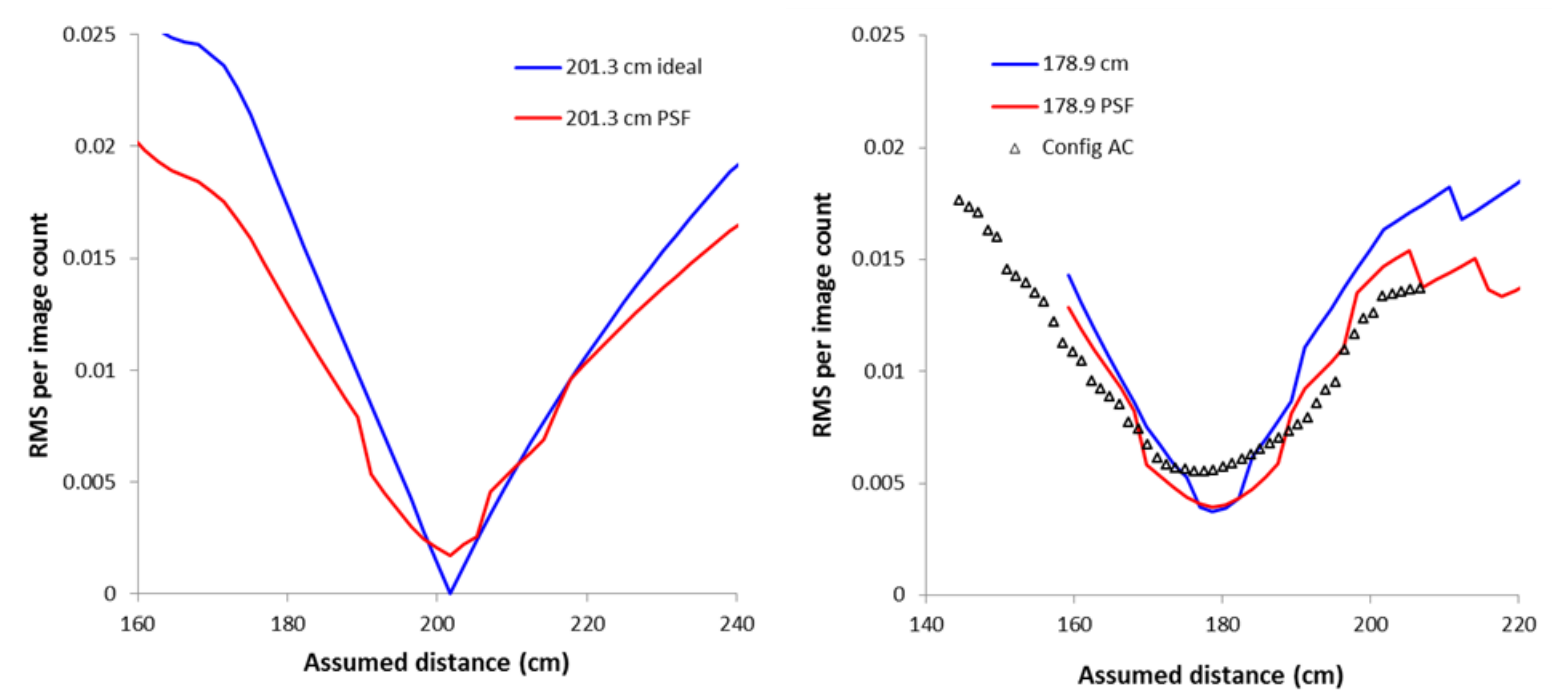

Fig. 13. The calculated net systematic error per image count for two configurations. Left, a configuration where the projection of the mask base pattern aligns with detector pixels boundaries and, right, the measured configuration.

Another significant source of systematic error originates in the propensity of neutrons to scatter in the detector and be recorded at locations different from the ones at which they were incident. The effect of scattering in the detector can be encoded in a point spread function (PSF) which characterizes probability of detection for a neutron as a function of distance from the position of incidence. The red curves in the plots of Fig. 13 show the slight decrease in sensitivity to changes in distance due to the point spread function. For comparison, the point source measurement data points are shown with black triangles in the plot on the right and agree rather well in both the depth and width of the minimum.

The distance to the source can be inferred from the data by finding the minimum in the systematic error. As an example, for the point source measurement, the minimum point has a value of 0.00563 and corresponds to a distance of $177.5 \mathrm{~cm}$. The true distance corresponds to $177.8 \mathrm{~cm}$. Measurement of the point source in several similar configurations with comparable statistics indicates that the repeatability of the distance measurement under these conditions is approximately $1 \mathrm{~cm}$. Similarly, estimation of the 
minimum point by means of fitting to a parabola introduces similar magnitude of error, and yields a minimum of $178.6 \mathrm{~cm}$. The parabolic fit to the point source data points (those shown by red triangles) is shown by the black line in the plot on the left in Fig. 14.
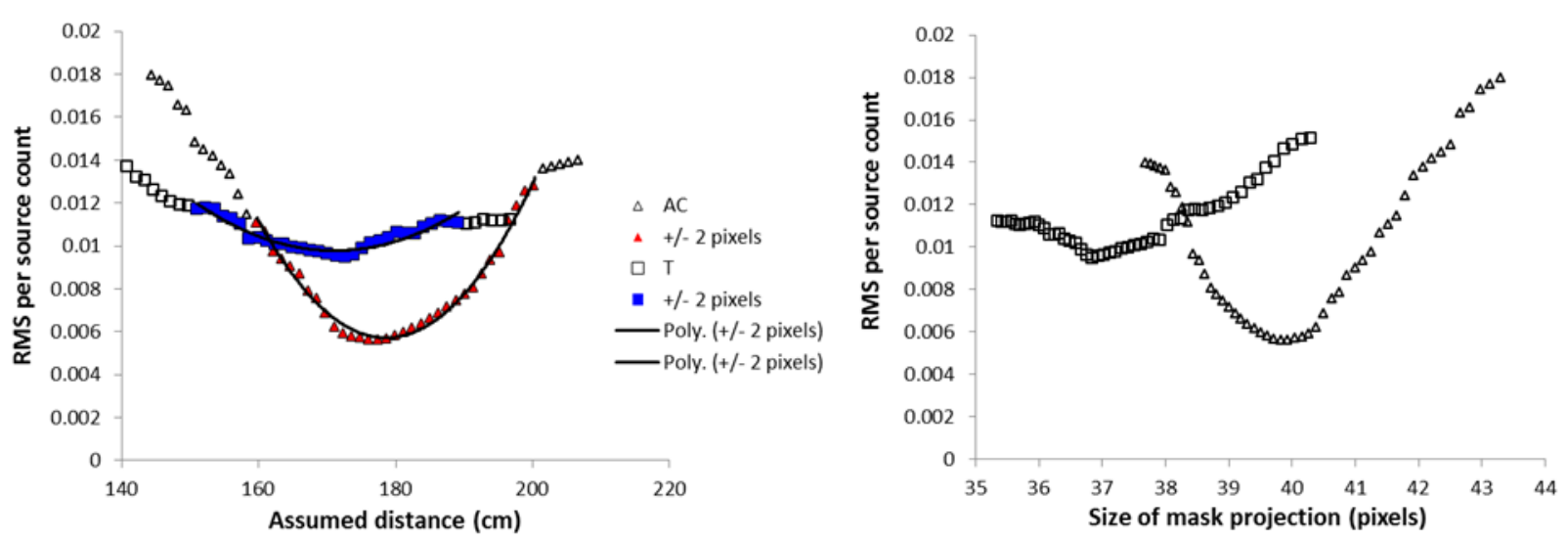

Fig. 14. Comparison of net systematic error per image count for a point source and an extended source. Left, this is plotted as a function of assumed distance, and right, it is plotted as a function of the size of the projection of the mask on the neutron detectors.

For comparison to the point source data, similar data is shown for an extended source. The extended source configuration is that shown in the center panel of Fig. 8. For a source that is extended in depth, the minimum systematic error increases because a fraction of the image counts are always out of focus. For a source that is extended in the imaging plane, the depth of the minimum is reduced in proportion to the square root of the number of pixels. For the example extended source measurement, which is extended in the imaging plane and in depth, the depth of the minimum in systematic error is four to five times less than for the point source. With similar errors on the individual points, it can be expected that the error in depth for the extended source measurements would therefore be a factor of four to five larger, and in fact, that is what is seen for repeated measurement of similar configurations.

It is also useful to look at the same plots of net RMS fluctuations per source count plotted as a function of the size of the mask projection. This plot is shown on the right of Fig. 14. Note that the position of the minimum for the point source (triangles) is known to approximately a tenth of a pixel and the position of the minimum for the extended source (squares) is known to approximately half a pixel. (It should be noted that additional work is necessary to subtract the baseline amount of fluctuations that is a function of the mask projections size. In the present analysis, this has not been done.)

In order to be able to predict the error in distance for other measurements, the accuracy of the determination of the minimum in net systematic RMS error per source count will scale like the width of the minimum divided by its depth in units of the error of the individual points. As such, the position resolution will scale like the error associated with the value of net systematic RMS fluctuations per source count $\boldsymbol{\alpha}$. Using rules for the propagation of error,

$$
(\Delta \alpha)^{2}=\left(\frac{1}{2} \frac{\Delta N_{T}}{\alpha N_{I}^{2}}\right)^{2}+\left(\frac{\sigma \Delta \sigma}{\alpha N_{I}^{2}}\right)^{2} \stackrel{\Delta N_{T} \text { small }}{\longrightarrow}\left(\frac{\sigma \Delta \sigma}{\alpha N_{I}^{2}}\right)^{2} .
$$

Note that the error associated with the first term can be neglected. Using the definitions of the experimental RMS fluctuations $\sigma$ and its associated error $\Delta \sigma$, we get 


$$
\Delta \sigma=\frac{1}{2} \sqrt{\frac{2}{n_{p}-1}} \sigma=\frac{1}{2} \sqrt{\frac{2}{n_{p}-1}} \cdot \sqrt{\left(\alpha N_{I}\right)^{2}+N_{T}}
$$

Substituting back into the expression for $\Delta \boldsymbol{\alpha}$, we get

$$
\Delta \alpha=\frac{\sigma \Delta \sigma}{\alpha N_{I}^{2}}=\frac{1}{\alpha N_{I}^{2}} \frac{1}{2} \sqrt{\frac{2}{n_{p}-1}} \cdot\left(\left(\alpha N_{I}\right)^{2}+N_{T}\right) .
$$

Making the substitution $\boldsymbol{N}_{I}=\boldsymbol{f} \boldsymbol{N}_{\boldsymbol{T}}$, the relative error in $\boldsymbol{\alpha}$ is then

$$
\frac{\Delta \alpha}{\alpha}=\frac{1}{2} \sqrt{\frac{2}{n_{p}-1}} \cdot\left(1+\frac{1}{\alpha^{2} f^{2} N_{T}}\right)
$$

Using this relationship, it is possible to scale the observed error of existing measurements with total counts $\boldsymbol{N}_{T}$ to get the resolution in centimeters as a function of counts in the imaging peak. These curves are plotted in Fig. 15 for the extended source (blue) and point source (red) configurations whose position resolutions were analyzed earlier.

Using the relative emission rates of ${ }^{252} \mathrm{Cf}$ and commercial reactor-grade $\mathrm{PuO}_{2}$, the image counts that would be recorded from a 1 hour measurement of a $20 \mathrm{~g} \mathrm{PuO}_{2}$ source is indicated by the black line. Note that if that source were point like, there would be sufficient resolution to constrain the source to be within the filter box. However, if (as is more likely) the source is distributed over the filter, the position sensitivity after an hour-long measurement would not constrain the average position of the source better than process knowledge of the location of the center of the filter.

In addition, we can plot the relative uncertainty in neutron source strength due to counting statistics and due to the uncertainty in distance as a function of the mass of $\mathrm{PuO}_{2}$ for 1 hour measurements. In Fig. 16, these are plotted for the point like and extended source distributions. Note that for $\mathrm{PuO}_{2}$ masses greater than approximately $20 \mathrm{~g}$, the error is dominated by counting statistics. For masses smaller than $20 \mathrm{~g}$, the error is dominated by uncertainty in position.

Given that holdup masses in filters are often smaller than tens of grams, improved distance determination is warranted. Furthermore, these plots correspond to the favorable geometries for position determination (the mask-to-detector distance is large and the field of view is small). Likely imager configurations with larger fields of view and shorter mask-to-detector distances will result in much diminished position determination. Examples of less favorable geometries performed during the present work include the wide-angle measurements of the HEPA house as well as the measurements of the large L-duct shown in Fig. 17. For the L-duct measurements, the corresponding plots of net systematic RMS per image count are shown as a function of assumed distance on the left of Fig. 17 and as a function of the size of the mask projection on the neutron detector on the right of Fig. 17. Recall that "L-Duct D" corresponds to a source configuration that is distributed across the imaging plane but not in depth and "L-Duct A" corresponds to a source configuration that is distributed across the imaging plane and in depth. Note that the position of the minimum for the source distributed across the imaging plane and in depth is known to approximately a pixel corresponding to an uncertainty in distance of approximately $50 \mathrm{~cm}$. 


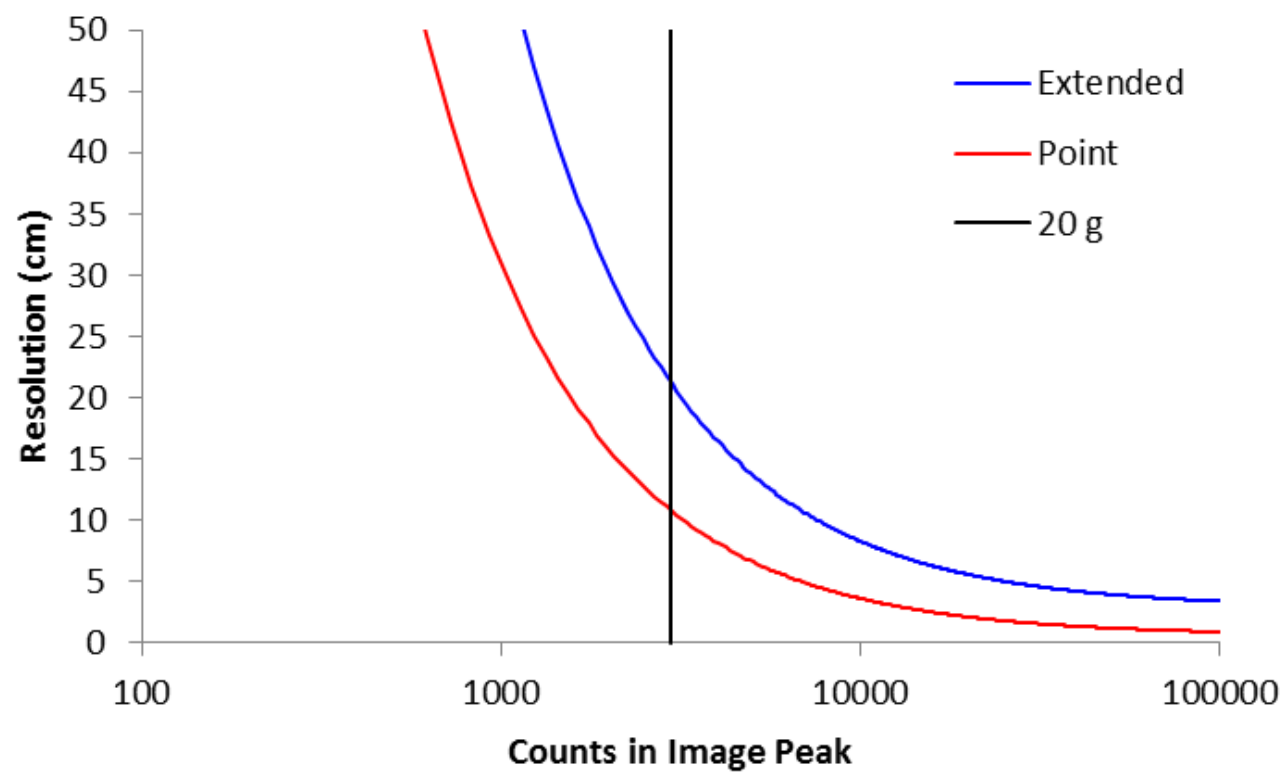

Fig. 15. Expected distance resolution for the zoomed point source and extended source configurations as a function of image counts. The vertical line indicates the number of counts expected for a 1 hour measurement of $20 \mathrm{~g}$ of commercial reactor-grade $\mathrm{PuO}_{2}$.

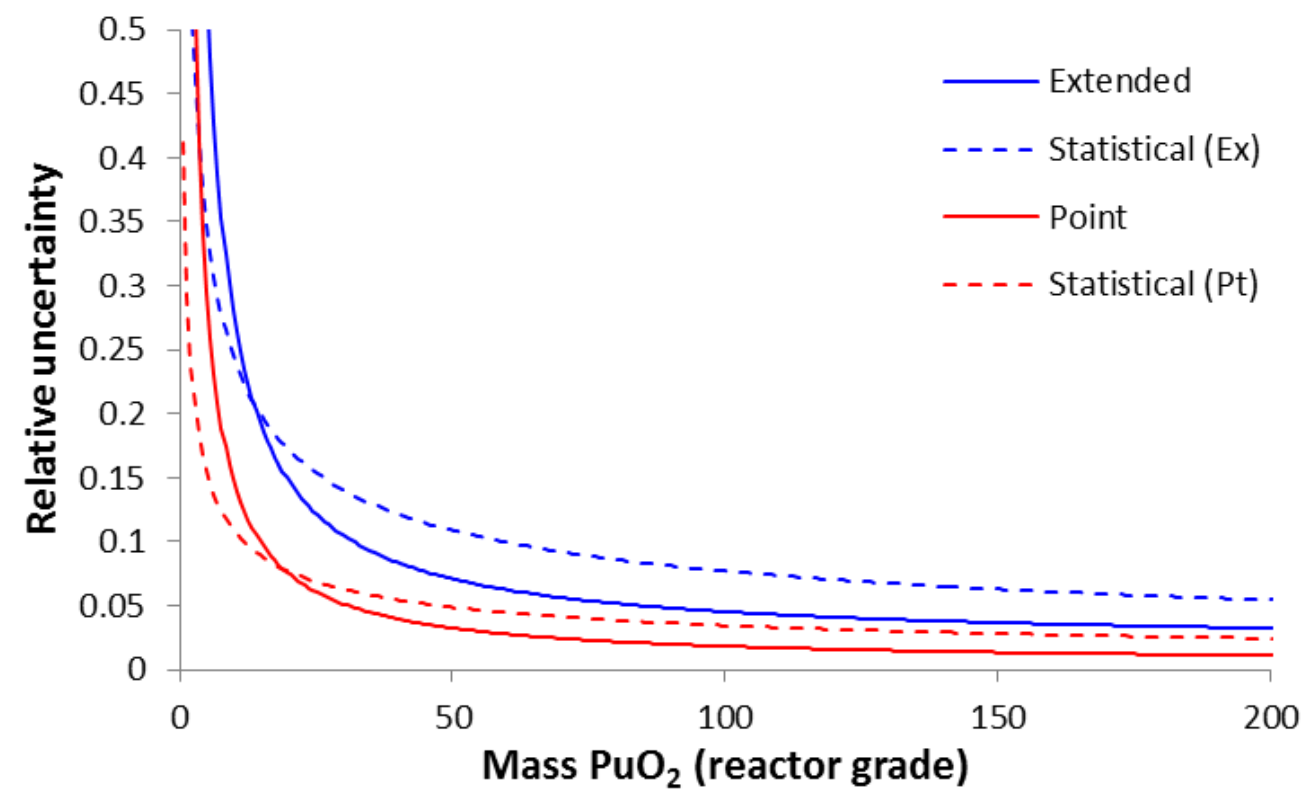

Fig. 16. Expected uncertainty in source strength for the zoomed point source and extended source configurations as a function of mass of $\mathrm{PuO}_{2}$. The solid lines indicate the contribution to error from uncertainty in distance, and the dashed lines indicate the contribution to error from counting statistics. 

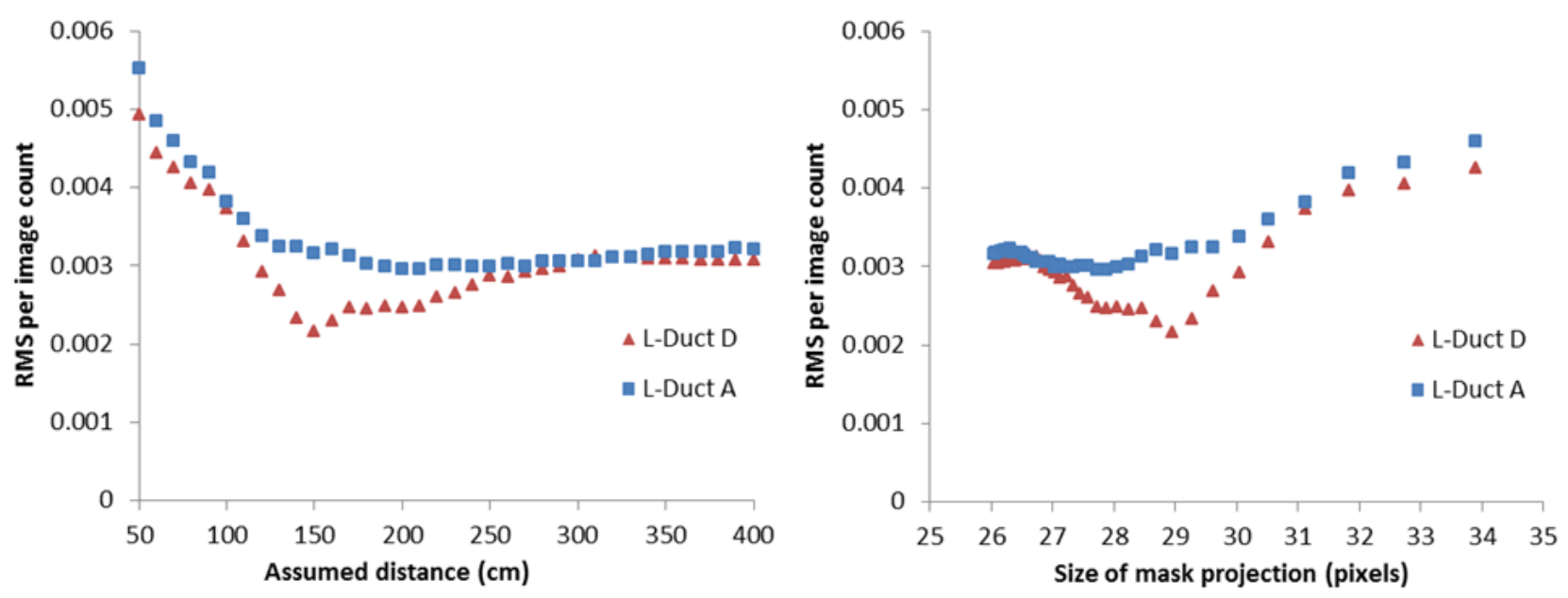

Fig. 17. Net systematic error per image count for two configurations of sources in the large L-Duct plotted (left) as a function of assumed distance and (right) as a function of the size of the mask projection on the detector.

The poor performance of the distance measurements in configurations where the imager has a wide field of view advocates for the use of position determination by the measurement of parallax. For the measurement of the point source with 65,000 image counts that achieved approximately 0.1 pixel uncertainty in the size of the mask projection and a corresponding uncertainty in source strength of 0.0085 , a similar uncertainty could be achieved using parallax with two measurements each having 3300 counts in the image peak. The combined counts of these two measurements would be 6600 , a factor of ten smaller than was necessary in the present measurements.

\section{CONCLUSIONS}

In the present work, sources of error that make quantitative neutron imaging measurements of holdup more difficult have been analyzed. While the shape of deposits contributes minimally to uncertainty, uncertainty in the location of the deposit contributes significantly. For most realistic amounts of holdup (grams or tens of grams of $\mathrm{PuO}_{2}$ ), the inherent distance resolution of the coded aperture technique imparted by measuring the size of the projection of the mask pattern onto the neutron detector panel is insufficient to make errors from distance uncertainty smaller than statistical errors. In addition, methods of measuring distance that are consistent with compact imager designs are desirable. By using parallax between multiple neutron imaging measurements, sufficient position resolution can be attained.

\section{REFERENCES}

[1] P. A. Hausladen, M. A. Blackston, and R. J. Newby, Position-Sensitive Fast-Neutron Detector Development in Support of Fuel-Cycle R\&D MPACT Campaign, ORNL/TM-2010/201 (2010).

[2] S. R. Gottesman and E. E. Fenimore, "New family of binary arrays for coded aperture imaging," Applied Optics, 28(20), 4344-4352 (1989).

[3] S. P. Cerne, O. W. Hermann, and R. M. Westfall, Reactivity and Isotopic Composition of Spent PWR Fuel as a Function of Initial Enrichment, Burnup, and Cooling Time, ORNL/CSD/TM-244 (October 1987).

[4] D. Reilly, N. Ensslin, and H. Smith, editors, Passive Nondestructive Assay of Nuclear Materials, pp. 339 and 345, NUREG/CR-5550 (March 1991). 